\title{
Digital radiography detectors - A technical overview: Part 1
}

\author{
Luís Lança $^{a, *}$, Augusto Silva ${ }^{b}$
}

a School of Health Technology, Lisbon Polytechnics, Lisbon, Portugal

b Department of Electronic, Telecommunications and Informatics, Aveiro University, Aveiro, Portugal

Received 14 February 2008; accepted 15 February 2008

\author{
KEYWORDS \\ $\mathrm{CR}$; \\ DR; \\ X-ray detectors; \\ Digital technologies
}

\begin{abstract}
During the last two decades screen-film (SF) systems have been replaced by digital $\mathrm{X}$-ray systems. The advent of digital technologies brought a number of digital solutions based on different detector and readout technologies. Improvements in technology allowed the development of new digital technologies for projection radiography such as computed radiography (CR) and digital radiography (DR). The large number of scientific papers concerning digital $X$-ray systems that have been published over the last 25 years indicates the relevance of these technologies in healthcare.

There are important differences among different detector technologies that may affect system performance and image quality for diagnostic purposes. Radiographers are expected to have an effective understanding of digital X-ray technologies and a high level of knowledge and awareness concerning the capabilities of these systems. Patient safety and reliable diagnostic information are intrinsically linked to these factors.

In this review article - which is the first of two parts - a global overview of the digital radiography systems (both CR and DR) currently available for clinical practice is provided. ( 2008 The College of Radiographers. Published by Elsevier Ltd. All rights reserved.
\end{abstract}

\section{Introduction}

Advances in digital technology allowed the development of full digital X-ray detectors that are currently available for projection radiography. Computed radiography (CR) and Digital radiography (DR) are digital technologies widely spread in healthcare institutions nowadays. These technologies have been replacing traditional screen-film (SF)

\footnotetext{
* Corresponding author.

E-mail address: luis.lanca@estesl.ipl.pt (L. Lança).
}

systems and this constitutes a challenge for radiographers and other healthcare staff.

The transition from a SF environment to a digital environment is not a simple matter. Technical factors concerning image acquisition, the management of patient dose and diagnostic image quality are some issues that could influence this process. In a transition process from SF to digital, patient radiation doses could increase $40-103 \% .{ }^{1}$ When compared to SF, digital technology could increase patient radiation doses due to the wide dynamic range they have. However, the dynamic range is useful because it contributes for a better clinical image quality when compared

1078-8174/\$ - see front matter @ 2008 The College of Radiographers. Published by Elsevier Ltd. All rights reserved.

doi:10.1016/j.radi.2008.02.004 
to traditional SF systems. ${ }^{2}$ This is an important difference among analogical and digital technologies. The risk of overexposure with no adverse effect on image quality could be present. Digital imaging systems could deliver over or under-exposure that influences patient's dose. Overexposure could provide good quality images, but may cause unnecessary patient dose.

The management of patient dose and the quality of images involves the relationship between three core aspects of the imaging process. ${ }^{3}$ These are determinants for the diagnostic quality of the radiographic image: (i) choice of radiographic technique; (ii) radiation dose to the patient and; (iii) diagnostic quality of the radiographic image. This is a challenge for radiographers because clinical advantages and limitations of digital technologies for projection radiography are also dependent on the radiographer's options for a particular patient examination.

A technical overview about digital radiography detectors is provided in this two part review article. In this article which is the first of two parts - a global overview of the digital radiography systems (both $C R$ and DR) currently available for clinical practice is provided.

\section{Overview of CR and DR detectors}

Several digital systems are currently available for the acquisition of projection radiographs. These digital systems are traditionally split into two broadly defined categories: computed radiography (CR) and digital radiography (DR). ${ }^{4,5}$ Although this taxonomy is commonly accepted other classifications are described: direct digital radiography and Indirect Digital Radiography technologies (including CR). ${ }^{6}$ In this case, the detector classification is related with the conversion process of X-ray energy to electric charge. Despite charge-couple devices (CCDs) could be considered indirect conversion DR systems, they are not flat-panel detectors and studies dealing with CCD-based digital general radiography are rare. $^{5}$ This technology is mainly related to other applications such as mammography and digital dental radiography. ${ }^{5}$ For this reason CCDs will be excluded from this review.

Despite the taxonomy that is used the major difference among digital technology systems is how the process of $X$ ray detection and readout is performed. Concerning $C R$ systems they use storage-phosphor image plates with a separate image readout process which means an indirect conversion process; DR technology converts X-rays into electrical charges by means of a direct readout process using TFT arrays. These systems can be further divided into direct and indirect conversion groups depending on the type of X-ray conversion used. ${ }^{5}$ Table 1 shows the differences among detector technology concerning three components of digital detectors ${ }^{7}$ : the capture element, the coupling element, and the charge readout element.

DR detectors can use either a direct or indirect process for converting $\mathrm{X}$-rays into electric charges. These detectors use direct readout by means of a Thin-film transistor (TFT) array despite the conversion process of the X-ray beam. Direct conversion detectors have a X-ray photoconductor such as amorphous selenium (a-Se) - that converts directly at only one stage X-ray photons into electric charges.

Indirect conversion systems use a two stage technique for conversion. They have a scintillator, such as Cesium lodide (Csl) that converts X-rays into visible light at a first stage. That light is then converted - at a second stage into an electric charge by means of an amorphous silicon photodiode array. ${ }^{8}$

CR technology uses an indirect conversion process using a two stage technique. X-rays are captured at a storagephosphor screen (SPS) (ex: $\mathrm{BaFBr}: \mathrm{Eu}^{2+}$ ) and then a photodetector captures the light emitted from the SPS and converts the captured luminescence into a corresponding digital image.

Fig. 1 show a general description model for digital X-ray technologies.

Despite the process of $\mathrm{X}$-ray detection and readout digital detectors offer several advantages when compared to SF systems. This includes wide dynamic range, adjustable image processing, better image quality, rapid image acquisition and image access at remote locations. ${ }^{9}$

\section{Computed radiography}

Computed radiography (CR) was the first available digital technology for projection radiography. CR technology is based in storage-phosphor screens (SPS) and its first clinical application by Fuji took place at the early 1980s.

This technology uses a photostimulable detector replacing the traditional SF cassettes. The storage-phosphor plates are exposed inside the cassettes with standard dimensions for typical plain radiography and no change of generator, X-ray tube and Bucky wall or table mounted system is necessary. CR technology allows the radiographer to obtain plain radiography images like in a traditional SF system. The difference is how the latent image is created and how this image processing is done. The basic CR imaging cycle has three steps ${ }^{6}$ : (i) expose, (ii) readout, and (iii) erase.

Inside the radiography cassette an image plate (IP) - or SPS - having a detective layer of photostimulable crystals is available. The detective layer consists of a family of phosphors BaFX:Eu ${ }^{2+}$ where $X$ can be any of the halogens $\mathrm{Cl}, \mathrm{Br}$ or I (or an arbitrary mixture of them). ${ }^{10} \mathrm{~A}$ typical SPS can store a latent image for a considerable period of time. However, it will lose about $25 \%$ of the stored signal

Table 1 Three components of digital detectors ${ }^{7}$

\begin{tabular}{|c|c|c|c|c|}
\hline \multicolumn{2}{|c|}{ Detector technology } & \multirow{2}{*}{$\begin{array}{l}\text { Capture element } \\
\text { BaFBr:Eu }{ }^{2+} \text { phosphor }\end{array}$} & \multirow{2}{*}{$\begin{array}{l}\text { Coupling element } \\
\text { Photostimulated luminescence (PSL) light-guide }\end{array}$} & \multirow{2}{*}{$\begin{array}{l}\text { Charge readout } \\
\text { Photo-multiplier tube; } \\
\text { signal digitization }\end{array}$} \\
\hline $\mathrm{CR}$ & & & & \\
\hline DR & Direct conversion & $\mathrm{a}-\mathrm{Se}$ & None & TFT array \\
\hline & Indirect conversion & $\mathrm{Csl}$ or $\mathrm{G}_{2} \mathrm{O}_{2} \mathrm{~S}$ phosphor & Contact layer & a-Si photodiode/TFT array \\
\hline
\end{tabular}




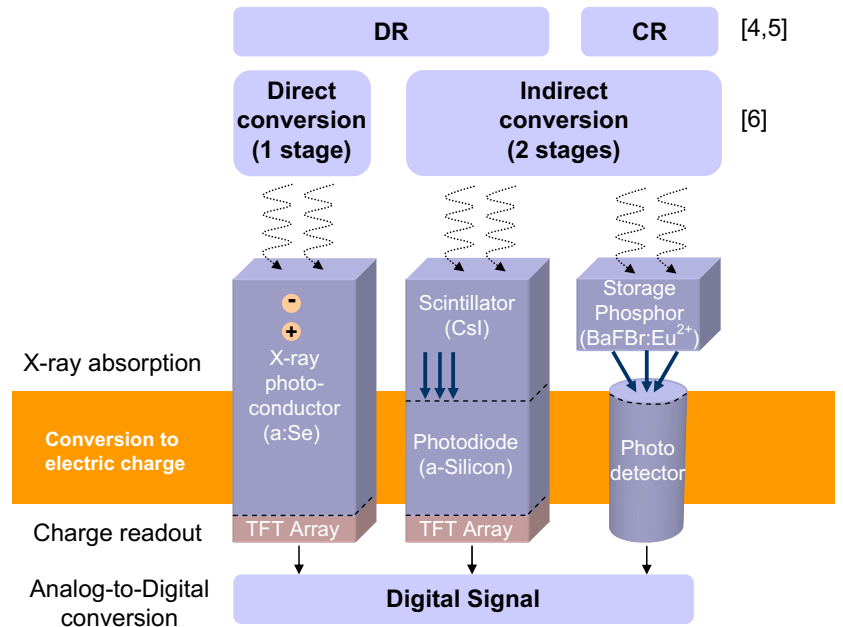

Figure 1 General description model for digital X-ray technologies (adapted from ${ }^{8}$ ).

between $10 \mathrm{~min}$ to $8 \mathrm{~h}$ after an exposure resulting in the loss of energy through spontaneous phosphorescence. ${ }^{11}$

The phosphor crystals are usually cast into plates into resin material in an unstructured way (unstructured scintillators). ${ }^{5}$ When the SPS is exposed to the X-ray the incident radiation excites electrons from the valence band to the conduction band (Fig. $2 a$ and $b$ ). These excited electrons absorb the X-ray energy and they are trapped at a stable energy level of the atom. The phosphor stores absorbed $X$-ray energy in crystal structure and a latent image is then created at these high-energy states giving a spatial distribution of these electrons at the SP detector. This trapped energy can be released if stimulated by additional light energy of the proper wavelength by the process of photostimulated luminescence (PSL). ${ }^{11}$

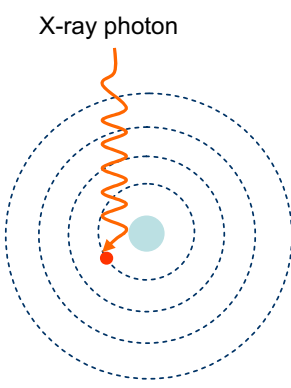

a

C

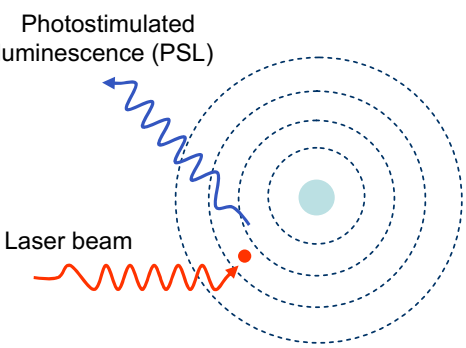

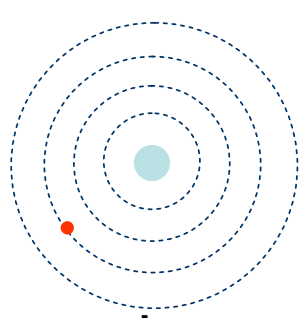

b
Figure 2 SPS exposure and PSL.
After the X-ray exposure and the creation of the latent image, the SPS is scanned in a separate CR reader device. The readout is a process that follows exposure of the image plate and constitutes the second step of the CR imaging cycle. A red laser beam scans the photostimulable screen stimulating the emission of blue light photons under the excitation of the laser beam. When the detective layer of the IP is scanned pixel by pixel with a high-energy laser beam of a specific wave length, stored energy is set free as emitted light having a wave length different from that of the laser beam. ${ }^{5}$ This triggers the process of photostimulated luminescence (PSL) resulting in the emission of blue light in an amount proportional to the original X-ray irradiation ${ }^{10}$ and setting free the excited electrons to their lower energy level (Fig. 2c and d). This light is collected by photodiodes and converted into electric charge while an analog-to-digital device converts it into a corresponding digital image. Fig. 3 shows the SPS scanning process.

Finally, the third step of the basic CR imaging cycle is the Residual Signal Erasure. Residual latent image electrons are still trapped on higher energy levels after readout. This energy is erased after the readout process using a highintensity white light source that flushes the traps without reintroducing electrons from the ground energy level. ${ }^{11}$

\section{Digital radiography}

Digital radiography (DR) flat-panel systems with integrated readout mechanisms were introduced in the market at the end of the 1990s. ${ }^{12}$ Flat-panel systems, also known as large area X-ray detectors, integrate an X-ray sensitive layer and an electronic readable system based on TFT arrays. Detectors using a scintillator layer and a light-sensitive TFT photodiode are called indirect conversion TFT detectors. Those using an X-ray sensitive photoconductor layer and a TFT charge collector are called direct conversion TFT detectors. ${ }^{12}$ The reference to amorphous silicon (a-Si), which is used in TFT arrays to record the electronic signal, should not be confused with amorphous selenium (a-Se) the material used to capture X-ray energy in a direct digital detector. The structure of a DR flat-panel system is shown in Fig. 4.

This electronic readable system allows an active readout process, also called active matrix readout, in opposition to the storage-phosphor systems where no active readout elements are integrated within the detector. The entire readout process is very fast, allowing further developments in digital real-time $X$-ray detectors. ${ }^{12}$

TFT arrays (Fig. 5) are typically deposited onto a glass substrate in multiple layers, with readout electronics at the lowest level, and charge collector arrays at higher levels. Depending on the type of detector being manufactured, charge collection electrodes or light sensing

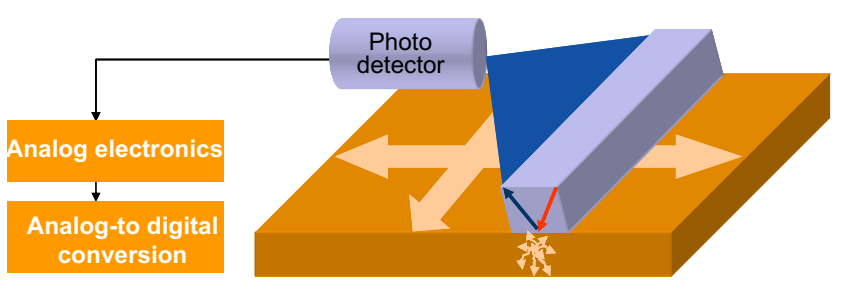

Figure 3 SPS scanning process. 


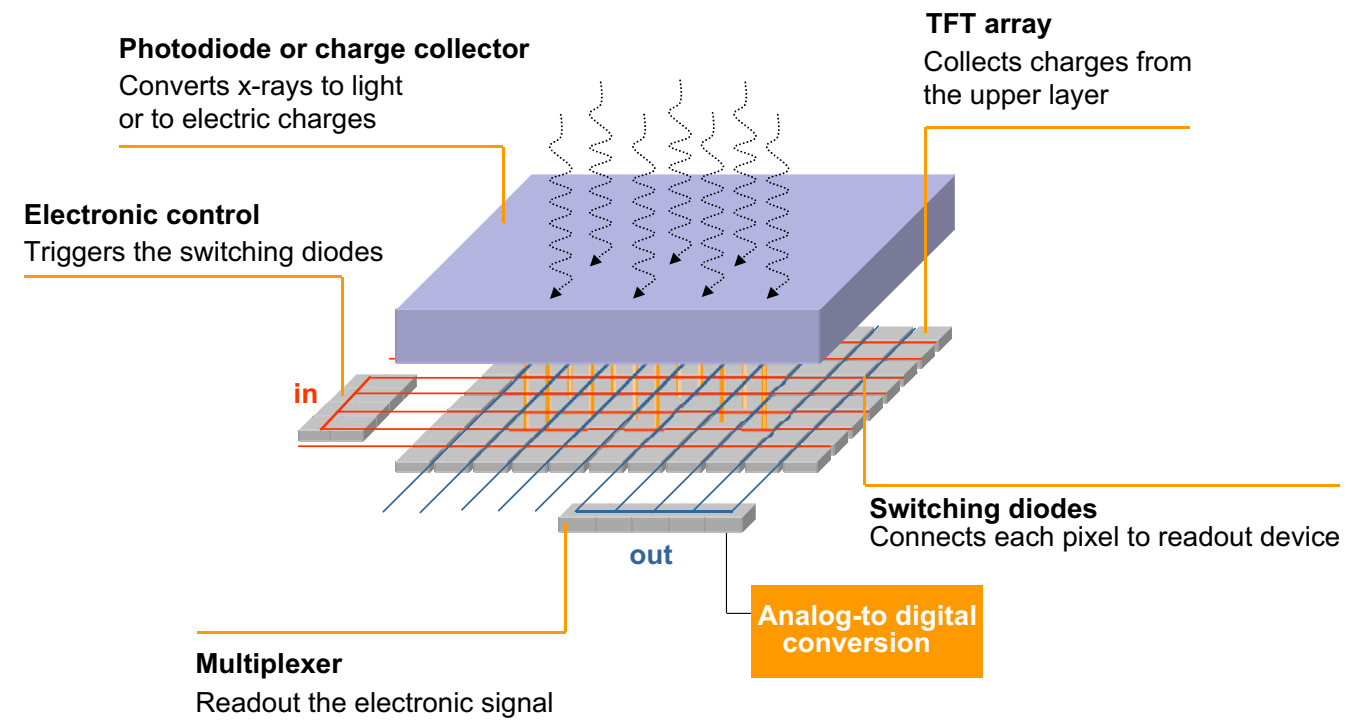

Figure 4 Flat-panel structure.

elements are deposited at the top layer of this "electronic sandwich.",13

The advantages of this design include compact size and immediate access to digital images. The performance of DR systems greatly exceeds the performance of CR systems, which have efficiencies of 20-35\%, and of screen-film systems for chest radiography, which have nominal efficiencies of $25 \%$. $^{13}$

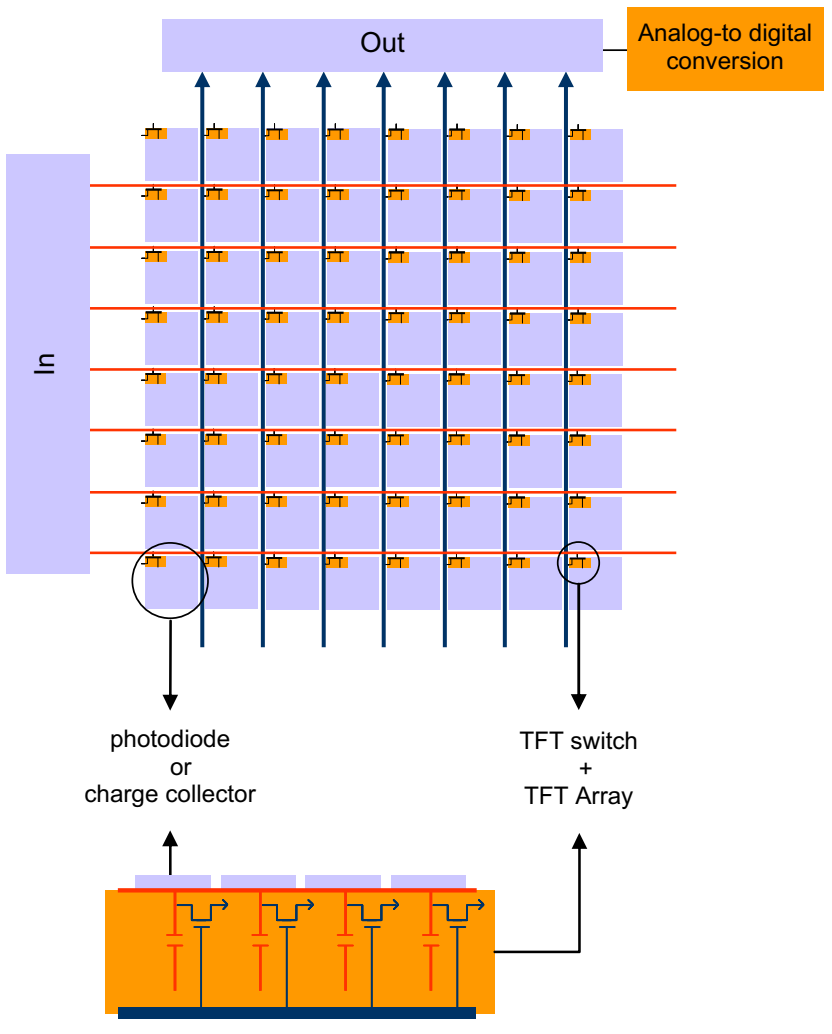

Figure 5 TFT array.

\section{Large area direct conversion systems}

Large area direct conversion systems use amorphous selenium (a-Se) as the semiconductor material because of its Xray absorption properties and extremely high intrinsic spatial resolution. ${ }^{12,13}$

Before the flat-panel is exposed to X-rays an electric field is applied across the selenium layer. Then the X-ray exposure generates electrons and holes within the a-Se layer: the absorbed X-ray photons are transformed into electric charges and drawn directly to the charge-collecting electrodes due to the electric field. Those charges proportional to the incident X-ray beam - are generated and migrate vertically to the both surfaces of the selenium layer, without much lateral diffusion. At the bottom of the a-Se layer, charges are drawn to the TFT charge collector, where they are stored until readout. The charge collected at each storage capacitor is amplified and quantified to a digital code value for the corresponding pixel. During the readout, the charge of the capacitors of every row is conducted by the transistors to the amplifiers.

\section{Large area indirect conversion systems}

Large area indirect conversion systems use cesium iodide (Csl) or gadolinium oxisulphide $\left(\mathrm{Gd}_{2} \mathrm{O}_{2} \mathrm{~S}\right)$ as an X-ray detector. The scintillators and phosphors used in indirect conversion detectors can be either structured or unstructured (Fig. 6). Unstructured scintillators scatter a large amount of light and this reduces spatial resolution. ${ }^{7}$ Structured scintillators consist of phosphor material in a needlelike structure (the needles being perpendicular to the screen surface). This increases the number of X-ray photon interactions and reduces the lateral scattering of light photons. ${ }^{7}$

When the scintillator layer is exposed to X-rays the beam is absorbed and converted into fluorescent light. At a second stage that light is converted into an electric charge by means of an a-Si photodiode array. ${ }^{8}$ Indirect conversion detectors are constructed by adding an a-Si photodiode circuitry and a scintillator as the top layers of the TFT sandwich. These layers replace the X-ray semiconductor 

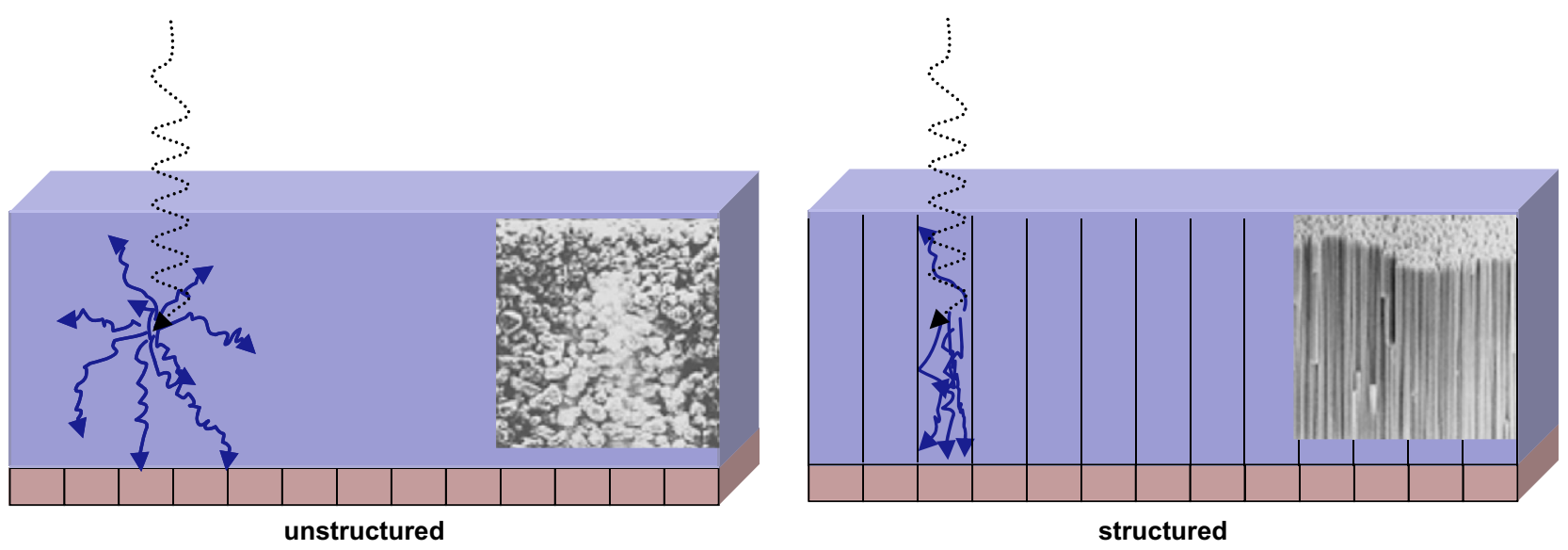

Figure 6 Unstructured or structured cintilator.

layer used in a direct conversion device. ${ }^{13}$ The active area of the detector is divided into an integrated array of image elements - the pixel - and each element contains a photodiode and a TFT switch available for the readout process.

\section{Conclusions}

Different digital technologies are currently available for projection radiography. DR and CR constitute a remarkable improvement in detector technology over the last 25 years. Although SF and digital technology coexist at the present time the trends in the future seems to point to the digital technology. The new digital and technological solutions at the radiography field seem to represent the change and the challenges concerning the radiographer's work in a digital environment. Further developments in digital detector technology are expected in the near future. This is an opportunity for the continuous improvement and optimization concerning the technical factors of image acquisition, the management of patient dose and the diagnostic image quality.

\section{Conflict of interest}

No conflict of interest is declared.

\section{References}

1. Vaño E, Fernández JM, Ten JI, Prieto C, González L, Rodríguez R, et al. Transition from screen-film to digital radiography: evolution of patient radiation doses at projection radiography 10.1148/radiol.2432050930. Radiology 2007;243:461-6.

2. Persliden J. Digital radiology and the radiological protection of the patient. Eur Radiol Syllabus 2004;14:50-8.

3. Busch $\mathrm{H}$. Image quality and dose management for digital radiography - final report. In: European Commission, editor. DIMOND3. Available from, <http://www.dimond3.org/European>; 2004.

4. Samei E, Seibert JA, Andriole K, Badano A, Crawford J, Reiner $B$, et al. AAPM/RSNA Tutorial on equipment selection: PACS equipment overview. Radiographics 2004;24:313-34.

5. Körner M, Weber CH, Wirth S, Pfeifer KJ, Reiser MF, Treitl M. Advances in digital radiography: physical principles and system overview. Radiographics 2007; 27:675-86.

6. Schaetzing R. Computed radiography technology. In: Advances in digital radiography: RSNA categorical course in diagnostic radiology physics. Chicago: RSNA; 2003. p. 7-22.

7. Samei E. Performance of digital radiographic detectors: factors affecting sharpness and noise. In: Advances in digital radiography. RSNA; 2003. p. 49-61.

8. Chotas HG, Dobbins III JT, Ravin CE. Principles of digital radiography with large-area, electronically readable detectors: a review of the basics. Radiology 1999;210:595-9.

9. Chotas H, Ravin C. Digital chest radiography with a solid-state flat-panel X-ray detector: contrast-detail evaluation with processed images printed on film hard copy. Radiology 2001;218: 679-82.

10. Rowlands J. The physics of computed radiography. Phys Med Biol 2002;47:R123-66.

11. American Association of Physicists in Medicine. Acceptance testing and quality control of photostimulable storage phosphor imaging systems. In: Report of AAPM task group 10. AAPM; 2006.

12. Kotter E, Langer M. Digital radiography with large-area flatpanel detectors. Eur Radiol 2002;12:2562-70.

13. Culley JD, Powell GF, Gingold EL, Reith K. Digital radiography systems: an overview. Hologic. Available from, <http://www. hologic.com/oem/pdf/DROverviewR-007_Nov2000.pdf >; 2000. 